\title{
Flexibilidad horaria, horas reales trabajadas y productividad laboral en el sur de europa
}

\author{
Alberto Vallejo-Peña \\ Universidad de Málaga \\ favallejo@uma.es \\ Sandro Giachi \\ Consejo Superior de Investigaciones Científicas-Universitat Politècnica \\ de València \\ sangia@upvnet.upv.es
}

Resumen: La sociología ha estudiado durante mucho tiempo la existencia de diversos modelos institucionales entre las áreas geográficas de Europa en términos de organización del trabajo. Sobre esta base, proponemos comparar la situación de cuatro paises representativos del sur de Europa (España, Italia, Grecia y Portugal) con la del resto del continente, centrándonos en el número de horas trabajadas y la flexibilidad horaria como parte de su modelo institucional de organización de la economía, así como su relación con los niveles de productividad laboral. Para ello, se han empleado indicadores de las oleadas de 2010 y 2015 de la Encuesta Europea sobre las Condiciones de Trabajo (EWCS, por sus siglas en inglés), que incluyen el número de horas trabajadas, la flexibilidad en las horas de entrada y salida y la tendencia a trabajar el mismo número de horas cada día. Tras comparar promedios en ambas oleadas $y$ aplicar regresiones lineales, se ha llegado a las siguientes conclusiones: la aplicación de fórmulas de flexibilidad horaria en Europa se asocia a altos niveles de productividad; $y$ los países del sur, aunque dedican muchas horas al trabajo, son menos productivos $y$ muestran una escasa flexibilidad horaria.

Palabras clave: países mediterráneos, economía en Europa, variedades de capitalismo, gestión del tiempo de trabajo, flexibilidad laboral. 


\begin{abstract}
Sociology has long studied the existence of various institutional models of work organization across Europe. On the basis of this, the present study compares the situation in four Southern European countries (Spain, Italy, Greece and Portugal) with that in the rest of Europe by examining the number of hours worked and working-hours flexibility as key elements in their institutional model for work organization, as well as the impact of the number of working hours on levels of labor productivity. The study uses indicators from the 2010 and 2015 editions of the European Working Conditions Survey (EWCS) which include the number of hours worked, working-hours flexibility, and the likelihood of working the same number of hours per day. After comparing averages in both surveys and applying linear regressions, we reached the following conclusions: 1) the application of flexible time policies in Europe is associated with high productivity levels; 2) although more hours are worked in Southern European countries, they are less productive and there is less flexibility regarding working-hours.
\end{abstract} Keywords: Mediterranean countries, European economy, varieties of capitalism, working time management, flexible working arrangements. 


\section{Introducción}

Desde un punto de vista histórico, Europa ha sido algo más que un continente: el territorio central que ha servido de eje a la sociedad occidental, generando el fenómeno político, cultural y económico conocido como eurocentrismo. Sin embargo, el dominio europeo ha ido cediendo gradualmente frente a regiones emergentes como los Estados Unidos - desde principios del siglo $\mathrm{xx}-\mathrm{y}$ el sudeste asiático durante las últimas tres décadas. Frente a la pujanza económica de estas zonas, Europa presenta síntomas de estancamiento (Lamo de Espinosa, 2010). Entre las evidentes diferencias económicas y culturales que existen dentro del continente, resultan destacables las dificultades de los países del sur para contribuir a la estabilidad económica y monetaria de la propia Unión Europea. Se trata de una cuestión compleja que implica múltiples factores y resulta difícil abarcar en una visión de conjunto. En el presente trabajo nos centramos en un subconjunto de factores específicos que podrían contribuir a explicar estos desequilibrios norte-sur en el continente. Dadas las demostrables diferencias en cuanto a productividad laboral entre los países mediterráneos y los países identificados como "del norte", indagaremos en un aspecto concreto como contribución al debate sobre las potenciales soluciones: la relación entre estas diferencias respecto al tiempo dedicado al trabajo y la gestión de la flexibilidad temporal. Para ello, nos sustentaremos en el enfoque de las variedades de capitalismo (Hall y Soskice, 2001) como principal referente teórico.

En términos teóricos, tomaremos como referencia la conceptualización de Eurostat, con la denominada productividad laboral por hora dedicada y persona empleada (Eurostat, 2018). Esta variable mide las unidades de producto generadas por cada hora trabajada. En realidad, las formas de productividad son diversas, por lo que dicha variable puede medirse en sentido más amplio si consideramos otros inputs al margen de las horas trabajadas (el gasto energético, por ejemplo). Sin embargo, dado el peso que la mano de obra tiene en las relaciones económicas, la anterior definición se ha impuesto sobre otras. Entrando en términos comparativos, el desempeño en productividad laboral de los países del sur de Europa ha evidenciado algunas debilidades en comparación con los países del centro y el norte. Siendo 100 el promedio de UE-28, Portugal y Grecia puntúan 68,3 y 67,2 respectivamente, mientras que Italia $(101,5)$ y España $(98,7)$ están muy cerca del promedio. Sin embargo, todos estos países están lejos de las puntuaciones de sus socios del norte, como Bélgica $(136,6)$, los Países Bajos $(129,4)$, Francia $(124,6)$ y Alemania $(123,0)$ (datos de Eurostat, 2015). Un informe del McKinsey Global Institute (MGI, 2015), que asigna 100 puntos al nivel actual de productividad laboral de los EE. UU., otorga 84 puntos al norte de Europa y 73 puntos a los 
países del sur de Europa. El informe indica en sus conclusiones que, aunque la productividad de Europa estuvo más cerca de la de los Estados Unidos durante la década de 1990, desde comienzos del nuevo siglo continúa disminuyendo, dada la presión a la baja de los países del sur.

Uno de los factores principales que se ha utilizado para explicar estas desigualdades se basa en la existencia de desequilibrios entre las necesidades productivas y el modelo ocupacional. En este sentido, Miguélez y Prieto (2009) destacan la gestación de un modelo laboral desequilibrado que frenó el progreso de la clase trabajadora. Mientras que el norte de Europa consolidó un modelo estable para el empleo durante el período 1950-1980, "los países del sur de Europa se quedaron atrás debido a la concentración de empleo de baja calidad y una deficiente inclusión de las mujeres en el mercado laboral" (Ibidem, 2009: 276). Además, a partir de las décadas de 1980 y 1990, este marco se debilitó por la emergencia de determinadas circunstancias que comprometen los derechos sociales: "En primer lugar, la fuerza del sistema de relaciones laborales de cada país, y luego la capacidad de inclusión dentro de cada mercado laboral de nuevos actores laborales diferentes al hombre adulto, como los jóvenes, las mujeres y los inmigrantes. [... En países como España, el dualismo del modelo laboral es muy evidente" (Ibidem, 2009: 276). Además, en los últimos veinte años el número de horas reales trabajadas no ha dejado de aumentar en los países mediterráneos. A esta tendencia histórica de sobrecarga de tiempo de trabajo se unió la crisis económica de 20082016, con los consecuentes recortes sociales al gasto público en Grecia, España y Portugal, lo que provocó una caída de los salarios y una pérdida de estabilidad de los contratos, en un contexto de negociación desequilibrado. Ello desembocó en una cultura de las relaciones laborales rígida y de cierta desconfianza entre gobierno, patronales y sindicatos (Leonardi et al., 2011). En este sentido, no se ha generado un clima institucional que facilite la implantación de la flexibilidad como dinamizador de la economía en los países del sur.

Es evidente, además, que esta dinámica del mercado laboral está relacionada con la composición sectorial de la economía en cada país. Por ejemplo, el caso de la agricultura, el turismo y los servicios en los países mediterráneos, frente a la industria y la alta tecnología en los países septentrionales. Los países cuyas economías están especializadas en las exportaciones y en industrias muy intensivas en conocimiento presentan, evidentemente, niveles mucho más elevados de productividad laboral en comparación con aquellos con otra especialización sectorial (Giachi y Vallejo, 2019). Además, debemos considerar las aportaciones de corte neoinstitucionalista, que subrayan que la configuración histórica del marco institucional de cada país se trasmite a la gestión de recursos humanos en el nivel 
de organización, aplicando distintas políticas de flexibilidad laboral. Este aspecto será tratado más extensamente en párrafos posteriores con aportaciones de Esping-Andersen (1999), Guillén (1994), Den Dulk et al. (2013), entre otros.

En definitiva, este conjunto de factores contribuye a explicar las desigualdades en cuanto a productividad laboral entre norte y sur de Europa. Como contribución particular, nos interesa un aspecto concreto dentro de estos procesos: la aplicación de políticas de flexibilidad horaria en el ámbito nacional y organizacional y su vinculación a la productividad laboral. Por lo tanto, expondremos los resultados de nuestra investigación sobre las diferencias de productividad laboral entre los países europeos y la influencia que en ella puedan tener los modelos nacionales de organización de los horarios de trabajo. Nuestro estudio focalizará particularmente su interés en el desempeño de los países más relevantes del sur de Europa (Italia, España, Portugal y Grecia). Utilizamos datos de la quinta (2010) y sexta (2015) olas de la Encuesta Europea sobre las Condiciones de Trabajo (EWCS), realizada por la Fundación Europea para la Mejora de las Condiciones de Vida y de Trabajo (Eurofound-Dublín). Para ello identificamos diversos indicadores relacionados con la flexibilidad de los horarios de trabajo como referentes de los modelos organizativos nacionales. Como punto de partida, establecemos la siguiente hipótesis de investigación: el modelo mediterráneo de capitalismo es menos productivo debido a la baja flexibilidad de la organización del tiempo de trabajo y los horarios, entre otros factores.

Abordamos, a continuación, los antecedentes teóricos que respaldan esta hipótesis (segundo apartado), la metodología (tercero), los principales hallazgos del estudio (cuarto) y las conclusiones (quinto).

\section{Variedades de capitalismo, organización del trabajo y gestión del tiempo}

El punto de partida de nuestra investigación se centra en la repercusión que el contexto nacional —en su faceta institucional y cultural - tiene en las prácticas organizativas y laborales de sus organizaciones y, por tanto, en la economía. Estas cuestiones han sido notoriamente analizadas por las ciencias económicas. La influencia del trabajo de Inglehart (1997), quien orientó sus estudios hacia el valor de la interculturalidad como elemento diferenciador entre países, fue crucial para focalizar las culturas nacionales. Asimismo, debemos destacar la investigación realizada por Esping-Andersen (1999), que conecta las culturas nacionales con el contexto sociopolítico. Este académico identificó tres modelos de desarrollo del Estado de bienestar en los países occidentales desde el final de la II Guerra 
Mundialः liberal, conservador y socialdemócrata. Más allá del sector público, todas las organizaciones (públicas o privadas) están integradas en dichos modelos.

Del mismo modo, debemos tener en cuenta las contribuciones del enfoque de investigación neoinstitucionalista. Mauro Guillén (1994), el autor de Models of Management, aborda estas diferencias según las relaciones económicas y laborales existentes internamente en cada país. La tesis de que las tradiciones de diferentes países marcan sus relaciones institucionales y su ideología es útil para explicar la mayoría de los conflictos organizativos y laborales existentes. De esta forma, se establecen dicotomías como "católico versus protestante" o "liberal versus conservador". En cada país, encontramos luchas institucionales que determinan el dominio de un determinado paradigma frente al otro, con las ineludibles repercusiones en los modelos organizativos desarrollados y en la productividad laboral.

Dentro de la literatura neoinstitucional, el enfoque de las "variedades del capitalismo" proporciona también una contribución relevante (Hall y Soskice, 2001). Estos estudios resaltaron la importancia de las instituciones nacionales dentro de la estructura económica, utilizando variables condicionantes como la innovación y el desempeño económico. Las variedades de modelos capitalistas en países y territorios se deben a razones históricas que, a su vez, tienen implicaciones en los sistemas de valores y creencias. El efecto institucional se manifiesta principalmente en los mecanismos de gobernanza de las relaciones laborales. Estos factores condicionan la capacidad de las naciones (y sus empresas) para adaptarse a los desafíos planteados por la globalización y el surgimiento de la economía del conocimiento (Hall y Soskice, 2001). La flexibilidad organizativa de los mercados y las empresas (por ejemplo, a través de nuevos modelos de relaciones laborales $\mathrm{u}$ organizaciones de red) destaca como un factor clave para fomentar la productividad y la competitividad de las empresas (Castells, 1997). En este sentido, hay países que han sabido adaptarse mejor a las nuevas formas de organización flexible y en red, frente a otros que han permanecido en trayectorias más "rígidas".

Este enfoque reconoce la existencia de diferentes tipos ideales de organización para la economía capitalista en Europa (Crouch, 1998). Por ejemplo, algunos estudios destacan la existencia de un modelo "mediterráneo" de capitalismo para países como Italia, España, Portugal y Grecia (Crouch, 1998; Amable, 2003). La idea de un modelo mediterráneo de capitalismo se basa en la existencia de grandes redes de pequeñas empresas basadas en la familia, la participación del gobierno en empresas mixtas y el papel destacado del Estado en la economía (De Jong, 1995; Moerland, 1995: 450-451; Regini, 1995), un sistema que De Jong (1995: 402) definió como "pragmático". En el caso mediterráneo, se dan determinadas 
razones históricas y culturales que explican por qué los países desarrollaron una forma peculiar de entender la organización, el trabajo y, sobre todo, la percepción del tiempo (Cassano, 1996).

Según Amable (2003), esta variedad de capitalismo es significativamente diferente al resto de modelos desarrollados en Europa. Por una parte, el liberal (o anglosajón) se basa en limitar la influencia de las instituciones sobre las empresas a través de mecanismos de regulación formal. Por otra, algunos países de Europa central (por ejemplo, Alemania) implantan una economía de mercado "corporativista", mientras que los países escandinavos adoptan el conocido modelo socialdemócrata. Estos dos últimos modelos destacan por su nivel de planificación a largo plazo de las políticas públicas nacionales y por promover estrategias de reclutamiento de las empresas más allá de las redes sociales cercanas.

Siguiendo líneas similares, cabe señalar los estudios internacionales comparados de Hall y Jones (1996), los que comparan las regiones canadienses realizados por Baldwin et al. (2005) y Maynard (2006), y el análisis de la economía española coordinado por Segura (2006). Tales aproximaciones muestran que las relaciones institucionales son clave para articular — de arriba abajo- los mecanismos económicos y organizacionales que facilitan la mejora de la productividad laboral, además de la capacidad tecnológica, la cualificación de la fuerza laboral y la internacionalización.

En este sentido, particularmente relevante resultó el estudio transnacional de Den Dulk et al. (2013). La apuesta de las instituciones por los patrones propios del Estado de bienestar tiene una repercusión evidente en la aplicación de fórmulas de flexibilidad laboral en el nivel organizacional (siendo más proclives a implementarlas). Si bien reconocen que esta trasmisión es mucho más notoria en las grandes organizaciones que en las pequeñas. Ambas circunstancias sitúan a los países del sur de Europa en una peor posición respecto a los del centro y el norte.

En definitiva, la literatura ha vinculado la productividad laboral con la existencia de valores, normas y reglas (el marco institucional) que facilitan la flexibilidad del sistema laboral y las consecuentes mejoras en la organización del trabajo (Esping-Andersen, 1999; Wall, 2007).

Estos antecedentes tienen una marcada repercusión en las pautas culturales dominantes en cada país, como es el caso de las diferencias existentes entre los horarios de trabajo de los países del norte y sur de Europa. Concretamente, mientras que las jornadas de trabajo en el norte terminan relativamente temprano (por lo general, alrededor de las 18:00 horas), en el sur resulta frecuente - en las empresas - apurar hasta bien entrada la noche, al menos en el caso de España y Portugal. Entre las explicaciones para la sobrecarga de tiempo de trabajo destacamos 
los estudios de Paugam (2008) y Halbwachs (2002), que reconocen la dificultad de las sociedades del sur para separar el tiempo de trabajo del tiempo privado o social. En cuanto al caso español, se puede profundizar con el trabajo de Moreno (2001): "La 'vía media' española del modelo de bienestar mediterráneo". El familismo, la importancia de las redes familiares y la "cultura de clan" (Banfield, 1958; Pizzorno, 1966; López Pintor y Wert, 1982) contribuyen a impedir la separación entre el trabajo y el espacio-tiempo privado. Estas cuestiones culturales se manifiestan claramente en los datos: Grecia, Italia y España ocupan los puestos $2^{\circ}$, $3^{\circ} \mathrm{y} 4^{\circ}$ del ranking internacional de países que más tiempo dedican a almuerzos y otras pausas durante los días laborables (OCDE, 2017), solo superados por los franceses. Todos ellos dedican diariamente un tiempo superior a dos horas a almuerzos o cafés.

Esta tendencia a la sobrecarga de tiempo de trabajo empeoró después de la crisis de 2008 dados los consecuentes recortes sociales al gasto público en Grecia, España y Portugal, lo que provocó una caída de los salarios en un contexto de negociación desequilibrada entre trabajadores y empresas privadas. A estas circunstancias se sumaron los aumentos de horas de trabajo obligatorias en el sector público en los países del sur y la limitación del número de trabajadores temporales empleados por la Administración pública (Barroso, 2017).

Entre las estrategias más relevantes promovidas en políticas públicas para fomentar la productividad laboral a través de la organización del trabajo se encuentran aquellas basadas en el tiempo flexible. Básicamente, se han abordado dos tipos de iniciativas: por una parte, la llamada flexibilidad pasiva (1), que evita que la empresa adopte los horarios de trabajo a las demandas y necesidades de los trabajadores al ofrecer beneficios como días libres o incentivos salariales (Lusa et al., 2007); por otra, las políticas de flexibilidad orientadas al personal (2), que fomentan su satisfacción y motivación a través de la mejora en la conciliación trabajo-familia (Jijena-Michel y Jijena-Michel, 2015; Lewis, 2003). En ambos casos, el apoyo percibido por el trabajador puede traducirse en actitudes positivas, con impactos significativos en la productividad, de acuerdo con la teoría del apoyo organizacional percibido y las teorías del intercambio social (Lambert, 2000). En este sentido, las políticas anteriores de flexibilización orientadas hacia el "cuándo" (se trabaja) se integraron con otras orientadas hacia el "dónde" y el "hasta qué punto" (Hill et al., 2008). De hecho, los estudios empíricos muestran que flexibilizar el sistema de horarios de trabajo de las empresas podría mejorar tanto la conciliación del trabajo familiar como la productividad laboral, generando a su vez reacciones positivas en los trabajadores, que tienden a mostrarse más satisfechos y productivos en algunos casos. Así lo dejó patente el clásico estudio 
de Wallace (2003), que comparó las nuevas formas de flexibilidad temporal en ocho países europeos, llegando a la conclusión de que estas fórmulas habían tenido un particular éxito en Reino Unido y Suecia. Posteriormente, investigaciones como la de Kossek y Van Dyne (2008) o Golden, Henly y Lambert (2014) en EE. UU., y la de Gordon, McMullin y Adams (2015) en Canadá, obtuvieron resultados similares. En cuanto a la experiencia española, un estudio reciente apunta en la misma dirección al analizar formas de promover la flexibilidad temporal a través del trabajo en equipo en la organización (Pinilla y López Peláez, 2017).

\section{Metodología}

El objetivo de esta investigación es medir la flexibilidad de los horarios de trabajo y su relación con la productividad laboral en los países del sur de Europa realizando una comparación con otras tres áreas previamente definidas por el enfoque teórico de las variedades de capitalismo. Para lograr este objetivo, se ha diseñado un estudio comparativo y exploratorio. A tal fin, se ha recurrido a datos del conjunto de países de la UE-28 sobre la base de datos de la EWCS (2010 y 2015). Por razones teóricas y operativas (usamos el promedio de los países europeos como referencia), nuestro diseño de investigación se centra en comparar el rendimiento de los siguientes cuatro bloques de países:

- Los cuatro grandes países del sur de Europa son representativos del modelo mixto (mediterráneo) de capitalismo: Italia, España, Portugal y Grecia. Estos países constituyen nuestro principal objeto de investigación y muestran niveles medio-bajos de productividad laboral.

- Los tres países del norte de Europa que siguen un modelo liberal (anglosajón) de capitalismo: Irlanda, los Países Bajos y el Reino Unido. Estos países muestran un nivel de productividad laboral superior al promedio.

- Los tres países del norte de Europa que siguen un modelo de capitalismo socialdemócrata (escandinavo): Dinamarca, Finlandia y Suecia. Estos países muestran también un nivel de productividad laboral superior al promedio.

- Los cuatro países de Europa central y occidental que siguen un modelo de capitalismo corporativista (continental): Austria, Bélgica, Francia y Alemania. Estos países muestran mayores niveles de productividad laboral.

Para la construcción de estos bloques, tomamos en cuenta las diversas áreas geográficas de Europa según la clasificación de las regiones naturales de la Organización de las Naciones Unidas (ONU; figura 1), junto con el esquema proporcionado por las variedades de literatura del capitalismo, según Hall y Soskice 
(2001) y la revisión realizada por Amable (2003). Este último autor especifica que la correspondencia geográfica de los modelos de capitalismo en Europa no ha de tomarse de forma literal, aunque resulta un referente práctico para los investigadores ${ }^{1}$.

Figura 1. Clasificación de Europa en regiones naturales (Naciones Unidas, 2018).

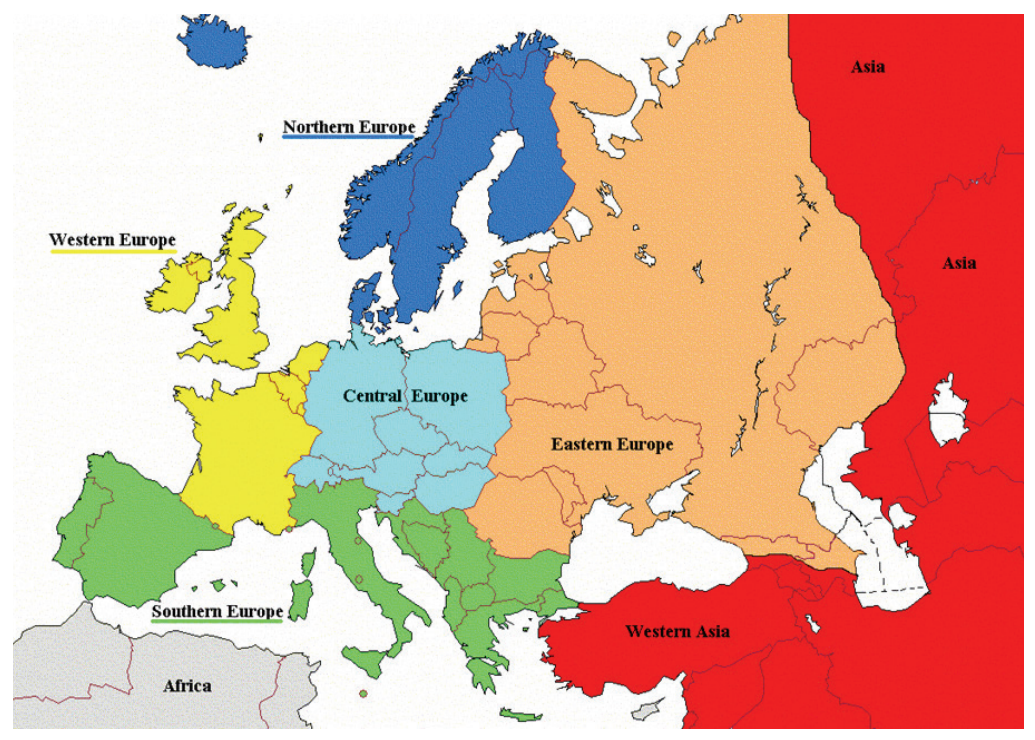

En primer lugar, presentaremos los datos recientes de productividad laboral de los países europeos para identificar así la situación de los cuatro bloques de países analizados respecto a este indicador (datos de Eurostat 2010 y 2015). En segundo lugar, tras analizar las diversas encuestas disponibles que relacionan el trabajo con los valores sociales, se ha optado por indicadores de la quinta y sexta oleada de la Encuesta Europea sobre las Condiciones de Trabajo (EWCS de Eurofound, 2010; 2015; véanse los datos técnicos en el anexo I). Finalmente, se ha recurrido como variables independientes a los siguientes indicadores, respec-

1 Aclaración de los autores: algunos países, como Chipre, Luxemburgo o Malta, no se han incluido entre los cuatro bloques indicados al no estar su modelo institucional claramente posicionado de acuerdo con la literatura. De hecho, no está claro si Chipre debe considerarse como un caso de la variedad liberal del capitalismo o mixto/ mediterráneo. Asimismo, los niveles desproporcionados de productividad laboral exhibidos por Luxemburgo, probablemente debidos a su estructura económica peculiar (finanzas internacionales), llevaron a la exclusión de este país de la clasificación. De igual forma, algunos países geográficamente correspondientes a la zona central y oriental de Europa (siguiendo el esquema de las Naciones Unidas) no se clasificaron entre los bloques definidos por su adscripción a la variedad poscomunista del capitalismo, al igual que ocurre con algunos países bálticos (Estonia, Letonia, Lituania). Estos presentan, en su conjunto, muy bajos niveles de productividad laboral. Sin embargo, los datos de todos estos países se han introducido dentro de la distribución europea para facilitar las comparaciones con el conjunto del continente. 
tivamente fundamentados en preguntas equivalentes del cuestionario empleado en ambas oleadas, que reportan el porcentaje de trabajadores encuestados que afirma:

- Trabajar menos de 30/34 horas por semana ("trabajadores sin sobrecarga").

- Trabajar más de 40 horas cada semana ("trabajadores sobrecargados").

- Tener horarios rígidos de entrada y salida en el trabajo ("horario de entrada/salida rígido").

- Trabajar aproximadamente el mismo número de horas todos los días ("horario de trabajo rígido").

Los primeros dos indicadores se refieren a la cantidad de tiempo empleado para trabajar, identificando el tamaño de dos grupos de trabajadores: por una parte, quienes trabajan semanalmente un tiempo moderado (menos de 30 horas en la encuesta de 2010 y menos de 34 en la encuesta de 2015); por otra, trabajadores sobrecargados, con más de 40 horas a la semana (indicadores 1 y 2 ). Asimismo, se aportarán los datos del promedio de horas trabajadas por semana según datos de la OCDE (2015). Por otro lado, se analizan dos variables que permitirán medir la gestión flexible del tiempo de trabajo, en este caso, a través de la medición del valor contrario: la rigidez del modelo organizativo relativa a los horarios (indicadores 3 y 4 ).

En resumen, analizaremos la relación entre las variables independientes identificadas con la productividad agregada a nivel nacional por hora trabajada (variable dependiente) según los datos de Eurostat en 2010 y 2015. Al cruzar las variables (en ambas oleadas), construimos un diagrama de dispersión y estimamos la pendiente lineal o polinómica, así como el coeficiente de determinación correspondiente. La fórmula estadística aplicada se describe a continuación.

Donde:

Yit $=$ Productividad por hora trabajada para el país $i$ en el año $t$

Xit $=$ Variable independiente (siendo $j$ la proporción de "trabajadores sin sobrecarga", "trabajadores sobrecargados" o "trabajadores con horario de entrada/ salida rígido" u "horario de jornada laboral rígido") para el país $i$ en el año $t$.

$K=$ constante del modelo.

El modelo se ha estimado siguiendo el procedimiento estandarizado de regresión de mínimos cuadrados, con apoyo de Excel.

En la presentación de los resultados, destacaremos la posición que ocupan los países mediterráneos en el gráfico de dispersión que pone en relación la cantidad de horas trabajadas y la flexibilidad de los horarios de trabajo (todas ellas 
variables independientes) con la productividad laboral de los respectivos países (variable dependiente).

Por otro lado, debemos resaltar que el Reino Unido ha sido tratado como país de la Unión Europea — a pesar de su reciente salida el pasado 30/01/2020_, ya que formaba parte de la UE en las fechas de las dos oleadas analizadas (2010 y 2015).

\section{Resultados}

En esta sección, exponemos los principales hallazgos del estudio. Primero abordamos la evolución reciente en la UE de los datos de productividad laboral por hora trabajada de los diferentes países (la variable dependiente del estudio). Tras esto se introducen los resultados de los análisis del impacto de las variables independientes seleccionadas sobre la mencionada productividad laboral.

\subsection{Productividad por hora trabajada en los países europeos}

La tabla 1 muestra los datos sobre los niveles de productividad laboral de los 28 países de la UE. Seleccionamos esta medida para la productividad considerando la relación entre el PIB y la cantidad total de horas trabajadas dentro del país. En este caso, los valores se han ponderado conforme al promedio de la UE-28, que se equipara a 100 para facilitar las comparaciones. Los países han sido clasificados de mayor a menor nivel de productividad, tomando 2015 como año de referencia (se aportan también los datos de 2010).

Tabla 1: Productividad laboral en la Unión Europea por países en 2010 y 2015 (considerando la media $=100)$

\begin{tabular}{|l|l|l|}
\hline País & 2010 & 2015 \\
\hline LU - Luxemburgo & 173,9 & 183,4 \\
\hline IE - Irlanda & 128,9 & 174,3 \\
\hline BE - Bélgica & 138,4 & 136,6 \\
\hline NL - Holanda & 132,2 & 129,4 \\
\hline DK - Dinamarca & 129,9 & 133,6 \\
\hline FR - Francia & 128,8 & 124,6 \\
\hline DE - Alemania & 126,1 & 123 \\
\hline AT - Austria & 113,5 & 119,5 \\
\hline SE - Suecia & 118,1 & 117,5 \\
\hline FI - Finlandia & 110 & 109,5 \\
\hline UK - Reino Unido & 104,8 & 101,8 \\
\hline
\end{tabular}




\begin{tabular}{|l|l|l|}
\hline País & 2010 & 2015 \\
\hline UE 28 - (28 países) & 100 & 100 \\
\hline IT - Italia & 103,7 & 101,5 \\
\hline ES - España & 99,5 & 98,7 \\
\hline MT - Malta & 75,9 & 80,3 \\
\hline SI - Eslovenia & 80,9 & 78,2 \\
\hline SK - Eslovaquia & 74,8 & 77,9 \\
\hline CY - Chipre & 77,6 & 76,2 \\
\hline CZ - Republica Checa & 69,1 & 74,5 \\
\hline PT - Portugal & 68,6 & 68,2 \\
\hline GR - Grecia & 75,2 & 67,3 \\
\hline HU - Hungría & 67,3 & 66,5 \\
\hline EE - Estonia & 60,7 & 64 \\
\hline LT - Lituania & 58,7 & 62,8 \\
\hline HR - Croacia & 56,4 & 62,8 \\
\hline PL - Polonia & 55,9 & 59,5 \\
\hline LV - Letonia & 49,6 & 55,4 \\
\hline RO - Rumanía & 43,7 & 53,7 \\
\hline BG - Bulgaria & 41,7 & 44,3 \\
\hline
\end{tabular}

Fuente: Eurostat (2015)

Al observar la tabla 1 , se aprecian pocas variaciones significativas en los niveles de productividad laboral de los países entre 2010 y 2015. Considerando la clasificación por regiones naturales, los países que muestran un nivel de productividad superior al promedio son parte del área occidental (Luxemburgo, Bélgica, los Países Bajos, Irlanda, Francia), a excepción del Reino Unido, seguidos por los países del norte (Dinamarca, Suecia, Finlandia) y dos países de Europa central (Alemania, Austria). Los países del sur de Europa ocupan posiciones que van desde el promedio de la UE-28 (Italia, España) hasta niveles más bajos que la media (Grecia, Portugal), junto con el Reino Unido, las islas mediterráneas (Chipre, Malta) y algunos países de Europa centrooriental (Eslovenia, Eslovaquia, República Checa). Las posiciones más bajas del ranking de la UE-28 están ocupadas por los países del este.

Por el contrario, si seguimos la clasificación de los países europeos según el esquema de variedades de capitalismo, observamos dos grupos de países en las posiciones de mayor productividad: el bloque "corporativista" (Bélgica, Alemania, Francia y Austria) y el bloque liberal, exceptuando el Reino Unido (Países 
Bajos e Irlanda). A estos les siguen de cerca el bloque socialdemócrata (tabla 1). A continuación, encontramos los países más grandes del bloque del sur (Italia y España), junto con algunos países del área anglosajona. Finalmente, encontramos a las otras dos economías mediterráneas en el ranking (Portugal y Grecia), con niveles de productividad muy próximos a los de las economías poscomunistas.

En resumen, las diferencias en el nivel de productividad laboral corresponden tanto a los patrones geográficos como a los modelos de capitalismo. Específicamente, podemos identificar un grupo sólido de países del noroeste de Europa formado por una serie de Estados que siguen patrones liberales o corporativistas y constituyen, de alguna manera, el "núcleo de la productividad europea". Por otro lado, los países nórdicos (socialdemócratas) muestran niveles próximos, aunque aún inferiores, a los dos grupos dominantes, y, finalmente, los países mediterráneos quedan lejos del "núcleo" y por debajo del promedio de la UE.

\subsection{Productividad laboral y cantidad de horas trabajadas}

El análisis del comportamiento nacional relacionado con la productividad laboral implica observar las diferencias en cuanto a la extensión de las jornadas laborales. Mostramos la relación existente entre los niveles agregados de productividad por hora trabajada de cada país (la variable dependiente) y el porcentaje de trabajadores sin sobrecarga en 2010 (gráfico 1) y 2015 (gráfico 2). Observamos que los países del sur de Europa muestran un porcentaje de trabajadores sin sobrecarga cercano al promedio: entre el $11 \%$ y el $18 \%$ para 2010 , y entre el $18 \%$ y el $27 \%$ para 2015 , con la excepción del caso de Italia para este año (algo más del 30\% de su plantilla).

Gráfico 1. Relación entre productividad y proporción de trabajadores que dedican menos de 30 horas (2010).

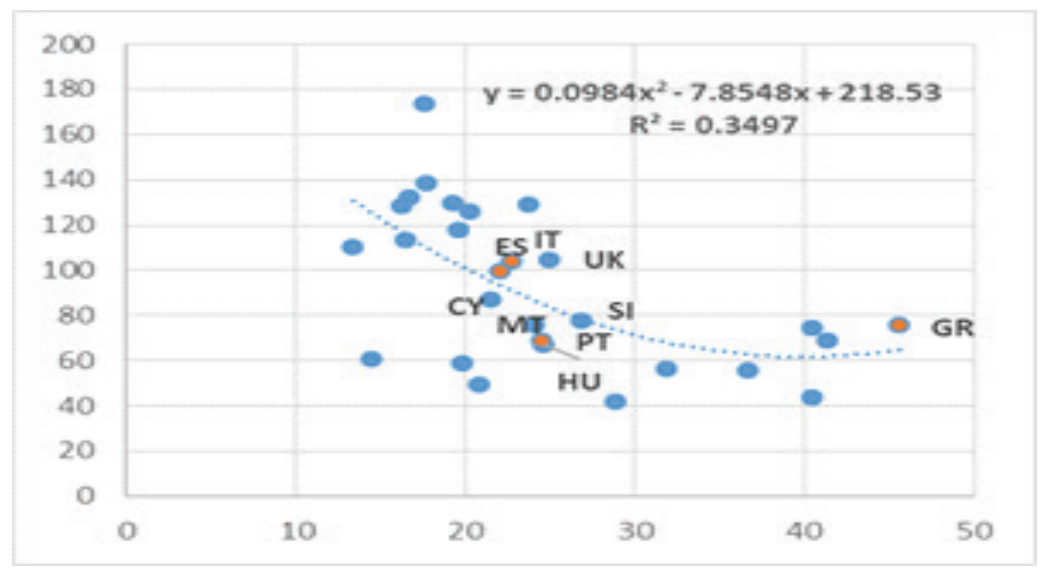


Gráfico 2. Relación entre productividad laboral y proporción de trabajadores que dedican menos de 30 horas (2015).

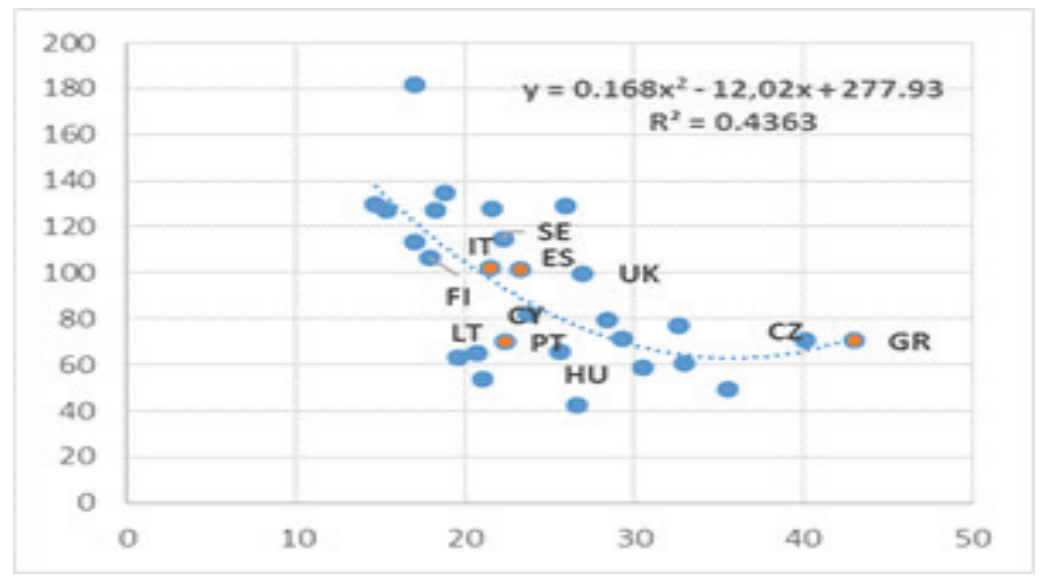

Tanto en 2010 (gráfico 1) como en 2015 (gráfico 2) observamos que existe una correlación significativa entre la productividad laboral y el porcentaje de trabajadores sin sobrecarga, como lo demuestra el coeficiente de determinación ajustado, superior a 0,5 en ambos casos. La relación entre ambas variables se ilustra mediante una pendiente polinómica positiva de segundo grado, que crece más rápido en correspondencia con los niveles más bajos de la variable independiente (trabajadores sin sobrecarga) y comienza a disminuir tras alcanzar el 30\% para 2010 y el $45 \%$ para 2015 (la diferencia se debe en gran medida a la forma de operacionalizar la variable). Esto significa que aumentar el porcentaje de trabajadores sin carga tiene un efecto positivo en la productividad, al menos hasta ciertos niveles. De hecho, todos los países que superan el promedio europeo (100) muestran un porcentaje de trabajadores sin sobrecarga superior a la media (13\% para 2010,18\% para 2015).

Los gráficos 3 y 4 muestran la relación entre los niveles de productividad agregada por hora trabajada y los porcentajes de trabajadores sobrecargados para cada país. La mayoría de los países del sur de Europa muestran niveles relativamente elevados para este indicador (entre $21 \%$ y $25 \%$ ), mientras que Grecia encabeza la clasificación en ambos años, con más del $43 \%$ de trabajadores sobrecargados sobre el total. 
Gráfico 3: Relación entre productividad laboral y proporción de trabajadores que dedican más de 40 horas (2010).

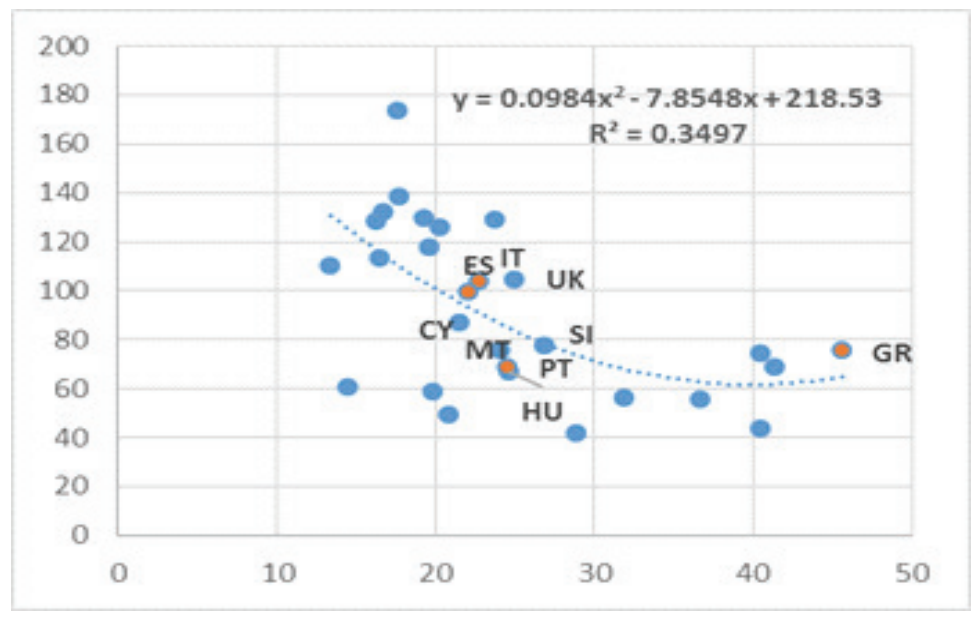

Gráfico 4: Relación entre productividad laboral y proporción de trabajadores que dedican más de 40 horas (2015).

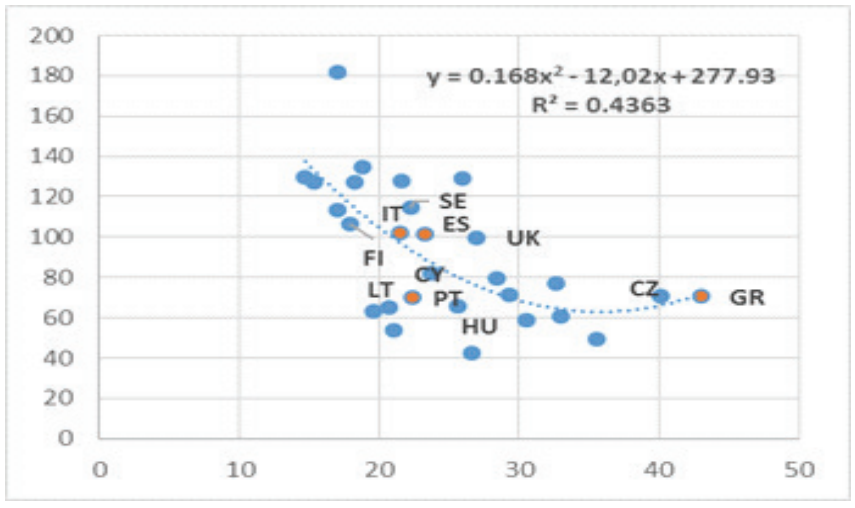

Tanto en 2010 como en 2015 observamos una correlación moderada entre la productividad y los trabajadores sobrecargados, ejemplificada por el coeficiente de determinación ajustado, superior a 0,34 para 2010 y superior a 0,43 para 2015. La relación entre estas dos variables se ilustra adecuadamente con la pendiente polinómica negativa, que desciende a mayor ritmo en correspondencia con los niveles más bajos de la variable independiente (trabajadores sobrecargados) y comienza a aumentar desde un umbral situado alrededor del 35\% de la distribución. Esto significa que aumentar el porcentaje de trabajadores sobrecargados tiene una relación negativa con la productividad, al menos hasta ciertos niveles. De hecho, todos los países que superan el promedio europeo (100) muestran un 
porcentaje de trabajadores sobrecargados por debajo de la media (entre el $25 \mathrm{y}$ el 27\%).

¿Qué relevancia tienen estos hallazgos para interpretar el desempeño del sur de Europa en cuanto a productividad laboral? La implicación más relevante es que la productividad por hora trabajada no parece aumentar con el número de horas trabajadas. Además, los países más productivos son aquellos en los que hay un porcentaje significativo de trabajadores sin sobrecarga (más de un tercio de la fuerza laboral total) y un porcentaje bajo de trabajadores sobrecargados (menos de una cuarta parte de la fuerza laboral total). Por otro lado, los trabajadores de los países mediterráneos pasan más tiempo trabajando que sus homólogos en los países liberales, corporativistas y socialdemócratas. Los países del bloque sur parecen luchar por aumentar sus niveles de producción a través del incremento de las horas extra.

De hecho, las horas reales trabajadas aumentan continuamente en los cuatro países mediterráneos analizados, presentando además un promedio superior a los países del norte (según datos de la OCDE 2015, véase gráfico 5). Dos de los cuatro países del bloque mediterráneo se encuentran entre los cinco países que más horas trabajan en Europa (Grecia ocupa el segundo lugar con 39,1 horas, solo superada por Polonia, y Portugal es quinto con 35,9), mientras que Italia y España presentan datos próximos a la media $(33,1$ y 32,5, respectivamente, siendo la media europea 32,6), aunque lejos de los valores de los países del norte. Esta sobrecarga horaria será difícil de controlar, dada su estrecha relación con aspectos culturales y estructurales que abordaremos posteriormente. Sin embargo, los países del norte presentan otro escenario: Dinamarca $(27,2)$, Noruega $(27,3)$ y Holanda $(27,4)$ lideran la clasificación de los países que menos horas trabajan. Los nórdicos destacan particularmente en esta clasificación (bloque socialdemócrata), pues todos ellos están entre los diez primeros de esta clasificación, con valores entre 27,2 y 31, salvo Islandia (36). Además, recientes análisis de los resultados de la European Work Conditions Survey-2015 nos muestran una rigidez horaria notoriamente mayor en los países mediterráneos en comparación con los del norte (Giachi y Vallejo, 2019). En definitiva, los países del sur presentan una sobrecarga horaria acompañada de una escasa flexibilidad horaria, lo que implica una carga adicional para el trabajador. Por el contrario, encontramos cuatro de los países más productivos del continente (Luxemburgo, Irlanda, Dinamarca y Finlandia) entre los cinco países que dedican menos tiempo a trabajar. 
Gráfico 5: Promedio de horas trabajadas a la semana en los países europeos (OCDE, 2015).

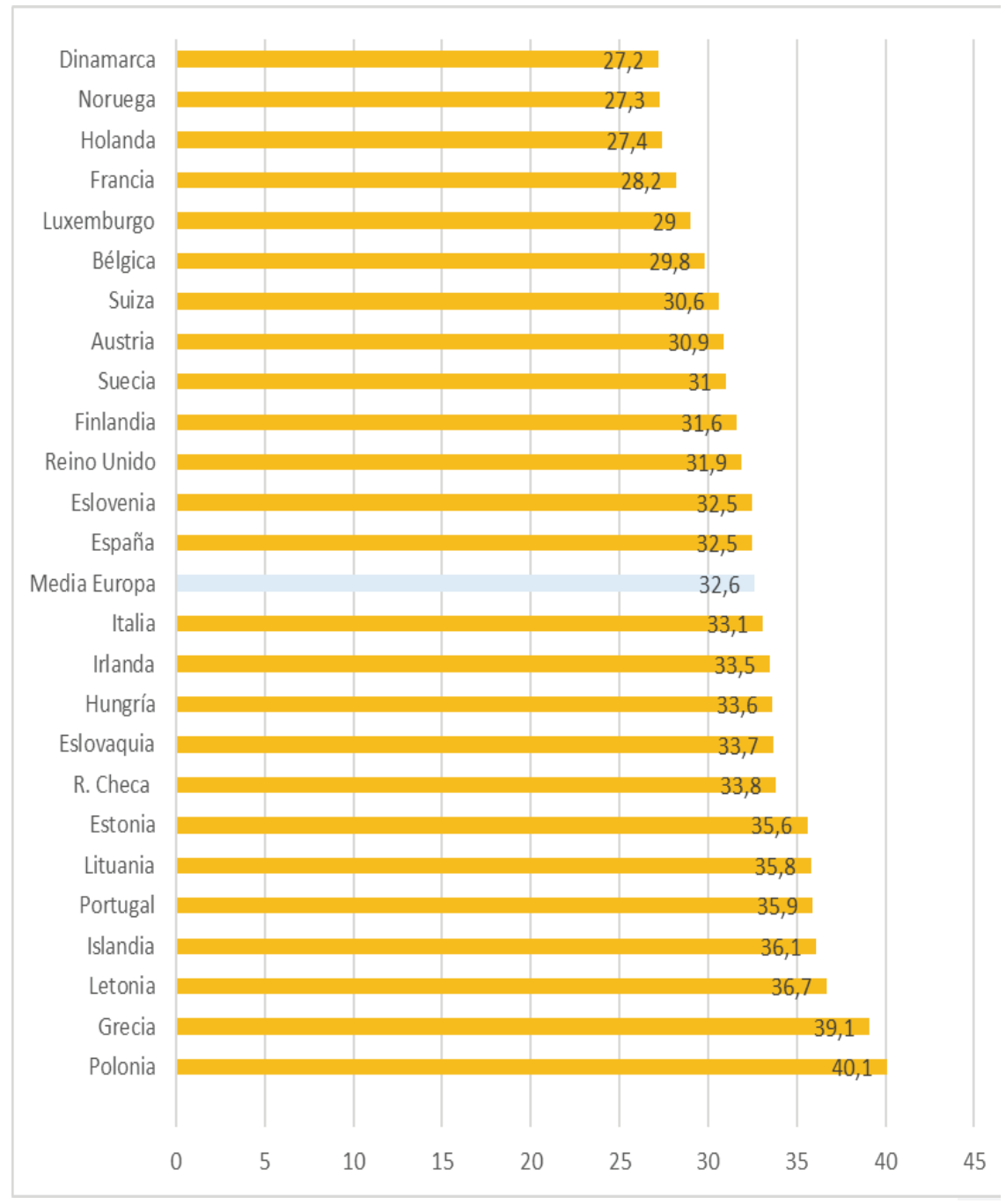

En resumen, la diferencia en términos de desempeño económico entre los países mediterráneos y los otros tres bloques está influenciada por un mayor número de horas dedicadas al trabajo de los primeros. En realidad, la reducción del tiempo de trabajo actúa como "recompensa" una vez que se logran determinados niveles de productividad. Tal relación parece particularmente evidente para los países del bloque socialdemócrata. Por el contrario, la productividad limitada de los países del sur provoca una extensión de la jornada de trabajo. Tales circunstancias sugieren que centremos nuestra atención en los factores culturales y 
organizativos que inhiben tal productividad. Concretamente, analizaremos hasta qué punto la productividad puede estar influenciada por factores que la teoría identifica como claves: la flexibilidad o rigidez de los horarios de trabajo.

\subsection{Productividad laboral y modelos organizativos flexibles}

Los gráficos 6 (2010) y 7 (2015) muestran la relación entre los niveles agregados de productividad por hora trabajada para cada país y el porcentaje de trabajadores con un horario de entrada/salida rígido. En ambas fechas, los países mediterráneos muestran una clara rigidez en los tiempos de entrada y salida en el trabajo en comparación con el resto de Europa, que exhibe valores superiores al $60 \%$, con porcentajes aún mayores para Portugal y España (más del $72 \%$ de su fuerza laboral total en 2010).

Gráfico 6. Relación entre productividad laboral y proporción de trabajadores con un horario rígido de entrada y salida (2010).

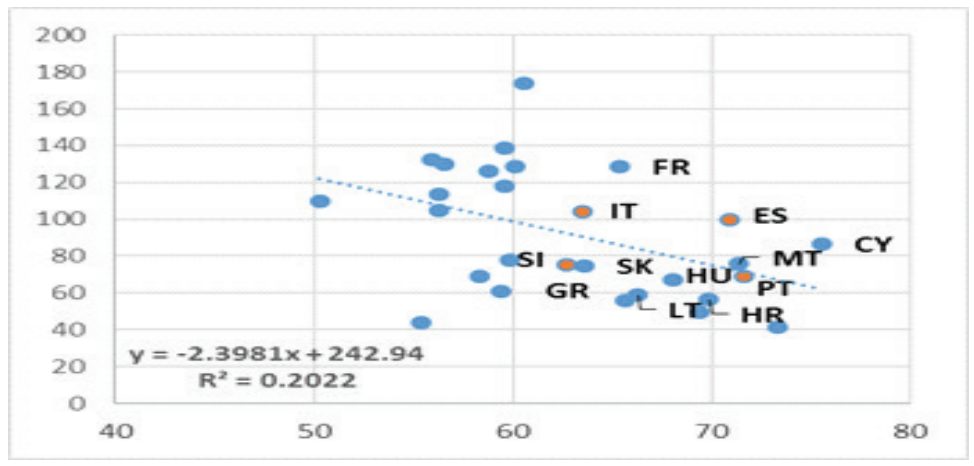

Gráfico 7. Relación entre productividad laboral y proporción de trabajadores con un horario rígido de entrada y salida (2015).

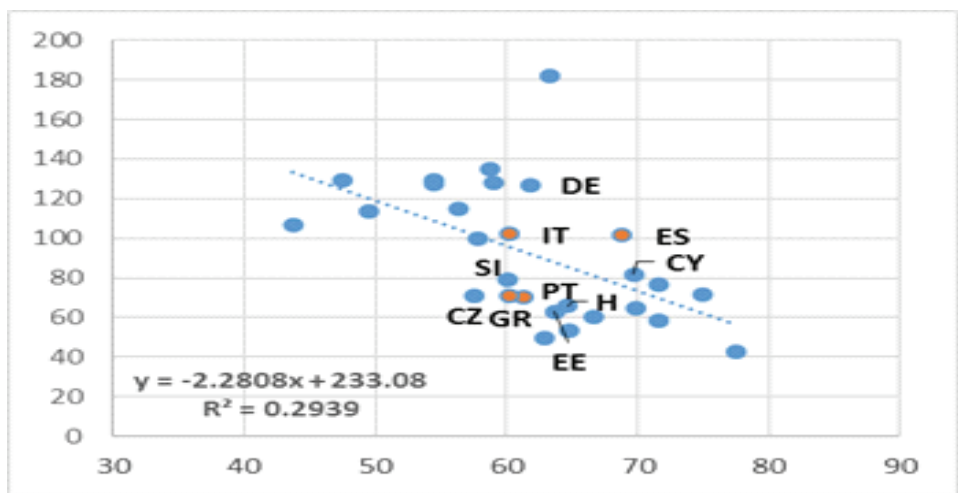


Observamos una correlación moderada entre la productividad laboral y el porcentaje de trabajadores con un horario de entrada/salida rígido. La relación entre las dos variables se expresa por una pendiente lineal negativa. Se estima un coeficiente de determinación de 0,20 para 2010 (gráfico 6) y 0,29 para 2015 (gráfico 7). Esto significa que los países con altos porcentajes de trabajadores con un horario de entrada/salida rígido muestran niveles más bajos de productividad laboral. Específicamente, observamos que todos los países con niveles de productividad más altos que el promedio europeo (100) presentan un modelo organizativo más flexible en términos de entrada/salida en el trabajo: sus porcentajes son inferiores al 60\% en la inmensa mayoría de los casos de ambas oleadas (2010 y 2015).

Los gráficos 8 (2010) y 9 (2015) muestran la relación entre los niveles agregados de productividad por hora trabajada para cada país y el porcentaje de trabajadores con horario laboral rígido. Se observa que los países mediterráneos presentan un mayor porcentaje de trabajadores con horario rígidoः más del $60 \%$ en todos los países del bloque y próximo al $70 \%$ en los casos de España y Portugal.

Gráfico 8. Relación entre productividad laboral y proporción de trabajadores con un horario de trabajo rígido (2010).

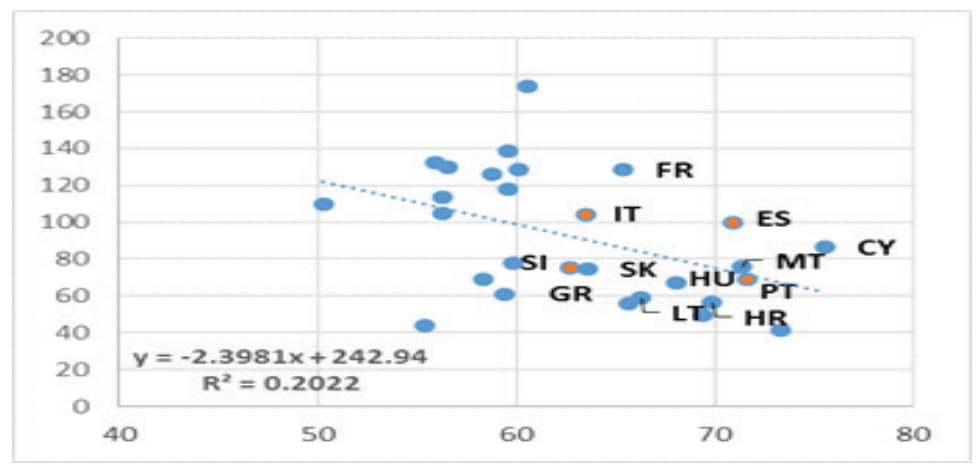


Gráfico 9. Relación entre productividad laboral y proporción de trabajadores con un horario rígido (2015).

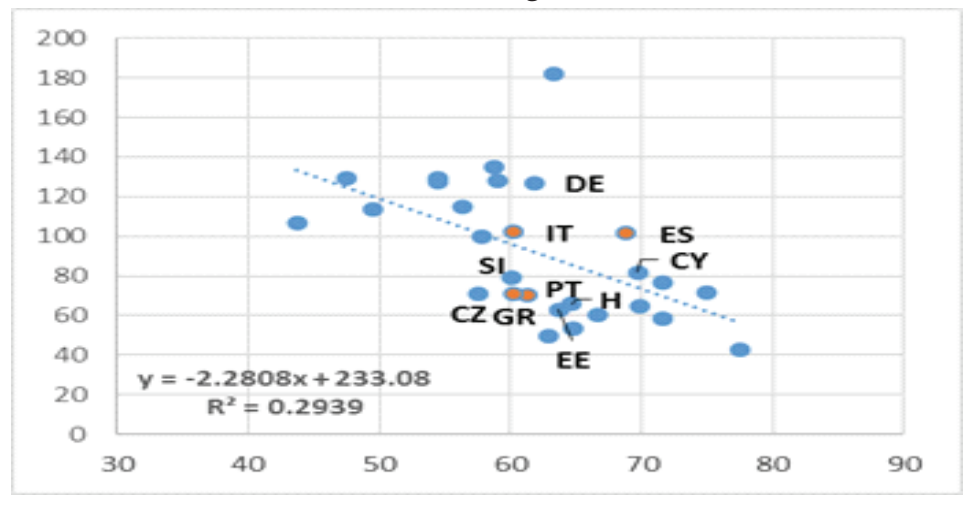

Se aprecia una correlación moderada entre la productividad laboral y el porcentaje de trabajadores con un horario laboral rígido. La relación entre las dos variables se ejemplifica por una pendiente lineal negativa. Se estima un coeficiente de determinación de 0,23 para 2010 (gráfico 8) y 0,35 para 2015 (gráfico 9). Esto significa que los países con horarios laborales rígidos muestran niveles más bajos de productividad laboral. Así ocurre con los países mediterráneos, que muestran valores superiores al 60\% tanto en 2010 como en 2015. Por el contrario, observamos que todos los países con niveles de productividad superiores a la media europea (100) presentan modelos organizativos más flexibles en términos de la gestión horaria (con valores inferiores al $60 \%$ en todos los casos).

En resumen, nuestros hallazgos muestran que los países europeos más productivos presentan una organización del trabajo orientada hacia jornadas más cortas y una mayor flexibilidad de los horarios de trabajo. En contraste, los países mediterráneos (Italia, España, Portugal, Grecia) tienen jornadas laborales más largas (aunque algo menos en el caso de Italia) y, sobre todo, un modelo organizativo más rígido respecto a la gestión del tiempo de trabajo. La brecha existente entre el bloque mediterráneo y las otras tres variedades de capitalismo identificadas queda manifiesta.

\section{Conclusiones}

En resumen, hemos clasificado los países europeos sobre la base de la clasificación regional propuesta por la ONU y la perspectiva de variedades de capitalismo (Hall y Soskice, 2001; Amable, 2003), identificando a los países europeos como países centrooccidentales (modelo corporativista), del bloque noroeste (liberal), norte (socialdemócrata) y sur (mediterráneo). A continuación, hemos compara- 
do los niveles de productividad por hora trabajada en las distintas áreas, siendo mayor en los primeros tres bloques, y destacando más aún en el reducido grupo de países corporativistas y socialdemócratas. En contraste, los países mediterráneos muestran niveles intermedios (España, Italia) o bajos (Grecia, Portugal) de productividad por hora trabajada.

Además, observamos que aquellos países con niveles más altos de productividad muestran promedios de horas trabajadas por semana — en general — bastante bajos, mientras que los países mediterráneos se sitúan por encima del promedio de la UE (salvo el caso italiano, levemente por debajo). En definitiva, los trabajadores del sur pasan más horas en el trabajo, pero tienen productividad - a nivel agregado - más baja. Esta cuestión se asocia a un desempeño laboral diferente durante la jornada laboral, más prolongada y con la interferencia de asuntos familiares o privados. Tales fenómenos tienen sus causas en aspectos estructurales, institucionales, organizativos y culturales, así como en la reciente adaptación a la crisis económica 2008-2015 (aspectos ya abordados en líneas anteriores).

Sobre la base de estos supuestos previos, realizamos los análisis de regresión descritos: el impacto de la gestión flexible del tiempo de trabajo sobre la variable dependiente productividad laboral, a través de los cuatro indicadores seleccionados como variables independientes (EWCS). Los resultados indican:

- Los países con una alta proporción de trabajadores sobrecargados (más de 40 horas) alcanzan más frecuentemente niveles bajos de productividad laboral.

- Los países con una alta proporción de trabajadores con jornadas de 34 horas o inferiores alcanzan más frecuentemente niveles altos de productividad laboral.

- Los países con una gestión flexible de los horarios de trabajo alcanzan más frecuentemente niveles altos de productividad laboral (sobre la base de los indicadores "tener horarios rígidos de trabajo durante la semana" y "tener horarios fijos de entrada/salida").

Asimismo, indagando en el desempeño del bloque mediterráneo, debemos destacar que se ha producido - en ambas oleadas - un corte estadístico entre España e Italia, por una parte, y Portugal y Grecia, por otra. La gestión organizativa de los dos primeros ha llevado a niveles moderados de productividad (en la media de la UE), a pesar de la distancia respecto a los países más productivos, mientras que en el caso de Grecia y Portugal es notablemente más baja (con valores alejados de la media de la $\mathrm{UE}$ ), aunque se dan indicios recientes de recuperación en el caso portugués. Esta circunstancia coincide con niveles más elevados 
en los dos indicadores de rigidez utilizados tanto en Portugal como en Grecia respecto a sus vecinos mediterráneos.

Los resultados del presente trabajo apuntan a favorecer, por los respectivos países, la aplicación de fórmulas flexibles en la gestión del tiempo para fomentar la productividad laboral. Debemos destacar que, en el caso particular de los países mediterráneos, la adopción de modelos organizativos más rígidos en la gestión de los horarios laborales contribuye al estancamiento de su productividad, tal y como se observa en los análisis de regresión realizados.

En términos globales, al valorar las futuras iniciativas políticas para equilibrar las economías mediterráneas, recomendamos que se consideren las funciones que deben cumplir las instituciones nacionales en la configuración de modelos organizativos flexibles, con particular énfasis en las que afecten a la gestión del tiempo de trabajo. Entre las diversas opciones de las instituciones, destacamos la flexibilidad en el tiempo de trabajo como estrategia. A medida que se superen las barreras institucionales, estructurales y culturales - que implican un esfuerzo adicional para el sur-, el fomento de tal flexibilidad puede contribuir a aumentar la productividad laboral en los países mediterráneos. A ello se suman los valiosos incentivos existentes para fomentar este cambio institucional y organizativo: mayor satisfacción de los trabajadores, una mejor conciliación familiar y la reducción de costes indirectos asociada al recorte de las jornadas de trabajo.

\section{Referencias bibliográficas}

Аmable, B. (2003). The diversity of modern capitalism. Oxford: Oxford University Press.

Baldwin, J. R.; Brown, W. M. y Maynard, J. P. (2005). Interprovincial Differences in GDP Per Capita, Labour Productivity and Work Intensity: 19902003, No. 2005011e, Statistics Canada, Economic Analysis.

Banfield, E. (1958). The moral basics of a backward society. Glencoe: The free press.

Barroso, M. (2017). "Crisis y trabajo. Un análisis de las políticas laborales de emergencia en Portugal, España y Grecia”. Revista Española de Investigaciones Sociológicas, 158: 3-22. (http://dx.doi.org/10.5477/cis/reis.158.3)

Cassano, F. (1996). Il pensiero meridiano. Roma-Bari: Laterza.

Castells, M. (1997). La era de la información. Madrid: Alianza.

Crouch, C. (1998). “Esiste una società europea?”. Stato e mercato, 2: 167-202.

De Jong, H. W. (1995). "European capitalism: Between freedom and social justice”. Review of Industrial Organization, 10(4):399-419. 
De la Fuente, A. (2005). "El impacto de la reducción de las ayudas estructurales europeas: una primera aproximación”. Presupuesto y gasto público, 39: 173-190.

Den Dulk, L.; Groeneveld, S.; Ollier-Malaterre, A. y Valcour, M. (2013). "National context in work-life research: A multi-level cross-national analysis of the adoption of workplace work-life arrangements in Europe". European Management Journal, 31(5), 478-494.

Esping-Andersen, G. (1999). Los tres mundos del Estado del bienestar. Valencia: Alfons el Magnànim.

Eurofound (2018). European Work Conditions Survey. Dublín. <http://www. eurofound.europa.eu>.

Eurostat (2018). Estadísticas oficiales de la Unión Europea. <https:// ec.europa.eu/eurostat/home?>

Giachi, S. y Vallejo Peña, A. (2019). "Cultural Differences in WorktimeFlexibility. A Comparison between Northern and Southern European Countries". 14th European Sociological Association (ESA) Conference, Manchester (RU): agosto, 20-23.

Gordon, C. E.; McMullin, J. A. y Adams, T. L. (2015). "Flexible small firms? Why some small firms facilitate the use of flexible workplace policies". Canadian Journal of Sociology/Cabiers Canadiens de Sociologie, 40(1).

Guillen, M. (1994). Models of management: work, authority, and organization in a comparative perspective. Chicago: University of Chicago Press.

Halbwachs, M. (2002 [1930]). Les causes de suicide. París: Coll. Le Lien Social. Hall, R. E. y Jones, C. I. (1996). The productivity of nations. $\mathrm{N}^{\circ}$ w5812, National Bureau of Economic Research.

Hall, P. A. y Soskice, D. (2001). Varieties of capitalism: The institutional foundations of comparative advantage. Oxford: Oxford University Press.

Hill,J.; Grzywacz,S.; Allen, V.; Blanchard, V;;Matz-Costa, C.; Shulkin, S. y Pitt-Catsouphes, M. (2008). "Defining and Conceptualizating Work Place Flexibility”. Community Work and Family, 11: 149-163.

Inglemart, R. (1997). Modernization and Postmodernization. Princeton University Press: Princeton.

Jijena-Michel, D. y Jijena-Michel, C. (2015).“El rol moderador de la flexibilidad del horario de trabajo en la relación del enriquecimiento trabajo familia y la satisfacción docente”. Horizontes Empresariales, 10 (2): 41-56.

Kossek, E. y Van Dyne, L. (2008). "Face-time matters: A cross-level model of how work-life flexibility influences work performance of individuals 
and groups". Handbook of work-family integration (305-330). Nueva York: Academic Press.

Lambert, S. (2000). "Added Benefits the Link Between Work-life Benefits and Organizational Citizenship Behaviour". Academy of Management Journal, 43: 801-815.

Lamo de Espinosa, E. (2010). Europa después de Europa. Academia Europa de las Ciencias y las Artes. Madrid.

Leonardi, L.; Martín, A.; Molina, O.; Calenda, D. y Carrasquer, P. (2011). “"Es exportable la flexiseguridad? Un estudio comparado de Italia y España”. Cuadernos de Relaciones Laborales, 29 (2): 417-443.

Lewis, S. (2003)."Flexible Working Arrangements: Implementations, Outcomes and Management". En C. Cooper e I. Roberts (eds.). Annual Review of Industrial Psychology, 18. Nueva York: Wiley.

López-Pintor, R. y Wert, J. (1982). “La otra España: insolidaridad e intolerancia en la tradición político-cultural española”. Revista Española de Investigaciones Sociológicas, 19: 292-307.

Lusa, A. et alii. (2007). “Gestión de los horarios de trabajo en presencia de cláusulas de flexibilidad pasiva”. Universia Business Review, 14 (2): 10-25.

Maynard, J. P. (2007). "The Comparative Level of GDP per Capita in Canada and the United States: A Decomposition into Labour Productivity and Work Intensity Differences". The Canadian Productivity Review, 15-206.

MGI (2010). Más allá de la austeridad: un camino hacia el crecimiento económico y la renovación en Europa. McKinsey Global Institute. <http://www.mckinsey. com/insights/mgi/research/productivity_competitiveness $>$.

Miguélez, F. y Prieto, C. (2009).“"Transformaciones del empleo, flexibilidad y relaciones laborales en Europa”. Política y Sociedad, 46 (2): 275-287.

Moerland, P. W. (1995). "Corporate ownership and control structures: An international comparison". Review of industrial organization, 110(4): 443-464.

Moreno, L. (2001). "La vía media española del modelo de bienestar mediterráneo". Papers, 63: 67-82.

OCDE (2019). Estadísticas oficiales de la OCDE. <https://stats.oecd.org/>

ONU (2019). "Mapas de Naciones Unidas". Organización de las Naciones Unidas. Nueva York. http://www.un.org/es/maps/.

Paugam, S. (2008). “Las formas elementales de la pobreza”. En Exclusión y desarrollo social en España. Madrid: Fundación Foessa.

Perrons, D. (2005). "Gender mainstreaming and gender equality in the new economy. An analysis of contradiction". International Journal of Politics, 7 (4): $389-411$. 
Pfirsch, T. (2013). "Une géographie de la famille en Europe du Sud". Cybergeo: European Journal of Geography, 533. <http://cybergeo.revues.org/23669>

Pinilla, F. y López Peláez, A. (2017)."La intensificación del trabajo en España (2007-2011): trabajo en equipo y flexibilidad”. Revista Española de Investigaciones Sociológicas, 160: 79-94. (http://dx.doi.org/10.5477/cis/reis.160.79)

Pizzorno, A. (1966). "Amoral familism and historical marginality". International Review of Community Development, 15: 55-66.

Regini, M. (1995). "La varietà italiana di capitalismo. Istituzioni sociali e struttura produttiva negli anni Ottanta". Stato e mercato, 43(2):3-21.

Segura, J. (ed.) (2006). La productividad en la economía española. Madrid: Centro de Estudios Ramón Areces. Madrid.

WALL, K. (2007). "Leave policy models and the articulation of work and family in Europe: a comparative perspective". International review of leave policies and related research, 2007, 25-43.

Wallace, C. (2003). "Work flexibility in eight european countries. A crossnational comparison". Czech Sociological Review, 21:773-794.

\section{Anexo Iः datos técnicos}

Encuesta Europea sobre las Condiciones de Trabajoः oleadas de 2010 y 2015. Datos técnicos (Eurofound, 2019)

Cobertura. 2010: 28 Estados miembros de la UE, Noruega, Suiza, Albania, antigua República Yugoslava de Macedonia, Kosovo, Montenegro, Serbia y Turquía. 2015: idéntica cobertura, exceptuando Kosovo.

Duración del trabajo de campo. 2010: enero a junio (todos los países incluidos). 2015: febrero a septiembre de 2015 en la UE-28, Noruega y Suiza; septiembre a diciembre de 2015 en Albania, antigua República Yugoslava de Macedonia, Montenegro, Serbia y Turquía.

Población objeto de estudio (2010 y 2015). Todos los residentes en los países mencionados anteriormente, de 15 o más años de edad (16 o más años de edad en Bulgaria, Noruega, España y el Reino Unido), y en situación de empleo en la fecha de la encuesta. Se consideró en situación de empleo a aquellos que hubieran trabajado a cambio de una remuneración o un beneficio durante un mínimo de una hora en la semana previa a la entrevista (definición de la OIT).

Muestreo (2010 y 2015): muestras aleatorias, estratificadas y multifásicas de la población activa en cada país. Dependiendo de la disponibilidad de registros de alta calidad, el muestreo se llevó a cabo utilizando registros individuales, de hogares o de direcciones, o a través del censo conforme a un procedimiento de recorri- 
do aleatorio. Las muestras de escala nacional se estratificaron por región y grado de urbanización. En cada estrato, se seleccionaron aleatoriamente unidades de muestreo primarias (UMP) de manera proporcional al tamaño. Posteriormente, se extrajo una muestra aleatoria de hogares en cada UMP. Por último, salvo en los casos en que se utilizaron registros individuales, el encuestado seleccionado en cada hogar fue la persona en situación de empleo cuyo cumpleaños fuera más próximo.

\section{Tamaño de la muestra:}

2010: en la mayoría de los países, el tamaño de la muestra objetivo se fijó en 1.000 entrevistas. Se aumentaron excepcionalmente las submuestras de Alemania y Turquía (2.000), así como en menor medida Italia, Polonia y Reino Unido (1.500). Eurofound brindó asimismo a los distintos países la posibilidad de ampliar su muestra. Tal oferta fue aceptada por Bélgica, Francia y Eslovenia, lo que dio lugar a tamaños muestrales de 4.000, 3.000 y 1.400 , respectivamente. El número total de entrevistas fue de 43.816.

2015: en la mayoría de los países, el tamaño de la muestra objetivo se fijó en 1.000. Con el fin de reflejar la existencia de una población activa más amplia en los países de mayor dimensión, tal tamaño se elevó a 1.200 en Polonia, 1.300 en España, 400 en Italia, 1.500 en Francia, 1.600 en el Reino Unido y 2.000 en Alemania y Turquía. Eurofound brindó asimismo a los distintos países la posibilidad de ampliar su muestra. Tal oferta fue aceptada por Bélgica, Eslovenia y España, lo que dio lugar a tamaños muestrales de 2.500, 1.600 y 3.300, respectivamente, en estos países. El tamaño total de la muestra en la sexta EWCS en el conjunto de los 35 países asciende a 43.850 entrevistas.

Tipo de entrevista (2010 y 2015): en persona, en el domicilio del encuestado; duración media: 45 minutos. 